\title{
A Beam-Steerable Antenna Based on the Spatial Filtering Property of Hyperbolically Anisotropic Metamaterials
}

\author{
Joshua K. H. Wong and Keith G. Balmain \\ University of Toronto, Toronto, Ontario, Canada
}

\section{Introduction}

The purpose of this work is to investigate the use of the spatial filtering property of hyperbolically anisotropic metamaterials to achieve beam-steering. A hyperbolically anisotropic metamaterial is a material whose dispersion surface is hyperbolic [1]. If a wave propagates within such a material, then the power flow will concentrate along the paths of zero reactance known as resonance cones [2] whose angle is a function of frequency [3]. Since the direction of power flow is frequency dependent, the phenomenon is also known as spatial filtering [4]. Spatial filtering has been used to achieve frequency diplexing [5]. In that context, different spectral components of a signal propagate along different directions in the metamaterial. However, the resonance cone angle is not only a function of frequency, but also a function of the permeability tensor of the metamaterial [1]. Thus, one can adjust the resonance cone angle by varying the elements in the permeability tensor, thereby varying the direction of power flow. The present work employs the aforementioned concept in a fixed-frequency antenna array. The radiation beam angle is made tunable by varying the permeability tensor of the metamaterial on which the antenna is realized.

\section{Theory of Operation}

A schematic of the antenna is shown in Fig. 1. The antenna is realized on a metamaterial based on [3] and consists of a square-celled grid situated above a ground plane and loaded with orthogonal series capacitors and inductors. The effective reactance of the series inductor is made tunable by loading it in series with two varactors as shown by the inset in Fig. 1. At each of the node crossings, a shunt inductor is connected to ground. This allows vertical currents to flow between the grid and the ground plane, which in turns allows vertically polarized radiation into the far field. Since the power flow in the grid concentrates along the resonance cone, the vertical currents will be strongest along the path of the cone. In this case, the radiation

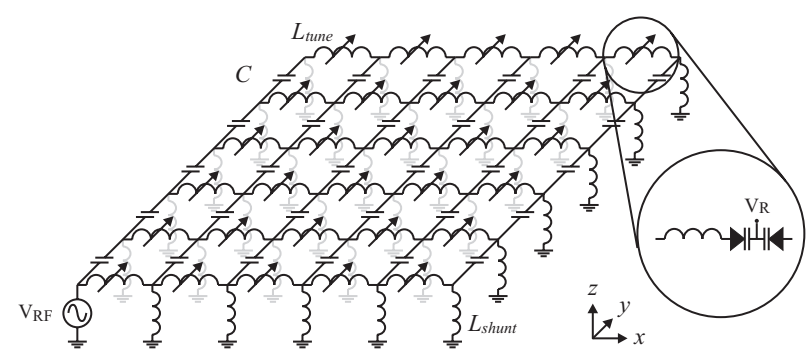

Figure 1: Schematic of the antenna. Not shown are the termination resistors along the top and right edges of the grid. The inset shows the implementation of the tunable inductors and how the varactors are biased. 


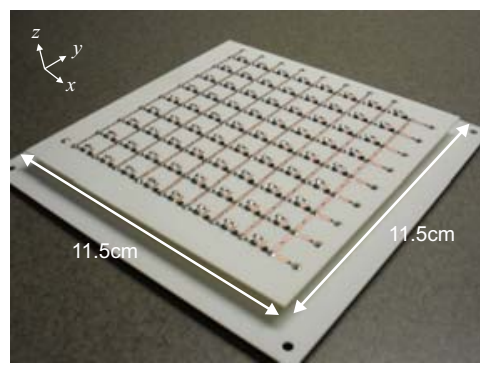

(a) perspective-view

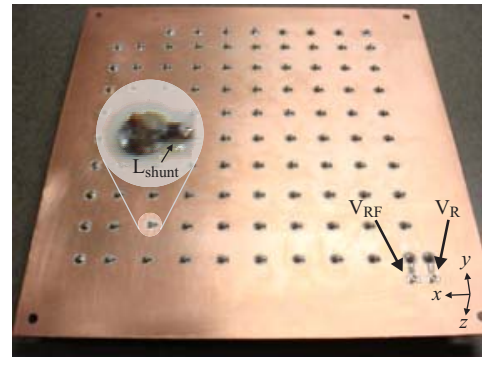

(c) bottom-view

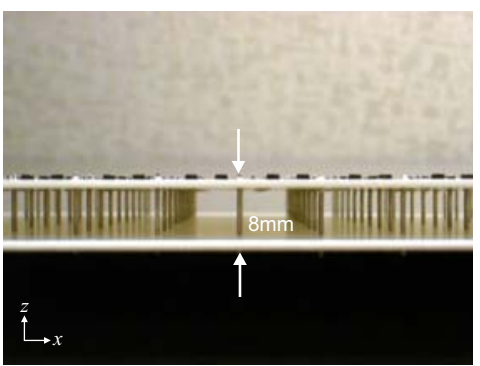

(b) side-view

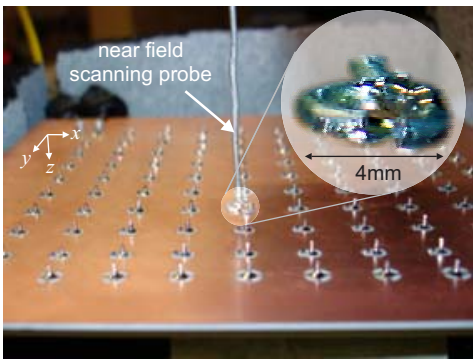

(d) near-field measurement setup

Figure 2: Photographs of the antenna prototype and the measurement setup.

pattern will have a main lobe oriented approximately broadside to the resonance cone in the azimuthal plane [6]. By varying the series inductance, the resonance cone angle changes and consequently the direction of the main lobe also changes.

\section{Experimental Results}

The completed antenna prototype is shown in Fig. 2. The design frequency of the antenna is $1.5 \mathrm{GHz}$. It consists of two dielectric substrates. The top side of the top substrate supports the anisotropic grid layer. This is the layer where the series capacitors, inductors and varactors are located as shown in Fig. 2(a). The series capacitance is $0.5 \mathrm{pF}$ and the tunable reactance is realized by a series inductorvaractor configuration. It consists of an $18 \mathrm{nH}$ inductor in series with a pair of varactor diodes. The diodes used in this antenna are Infineon BB831 with an upper operation frequency of $2.5 \mathrm{GHz}$. The DC biasing circuits are located on the bottom side of the top substrate. The biasing scheme is shown in Fig. 1. The cathodes of the varactor pair are connected opposite to each other. The ground plane is located on the bottom side of the bottom substrate. Metal pins, which also act as vias, were used to connect the top substrate together with the bottom substrate as shown in Fig. 2(b). In series with the pins are the $28 \mathrm{nH}$ shunt inductors as shown in Fig. 2(c). The DC bias voltage and the RF signal are fed into the antenna through two UFL connectors located on the ground plane layer as shown in Fig. 2(c).

The first step in characterizing the antenna is to verify that the resonance cone angle is tunable. A straightforward method to locate the resonance cone is to measure the vertical E-field above the grid [3]. However, the presence of a probe would introduce distortion to the near field since the antenna is radiating on the top side. 


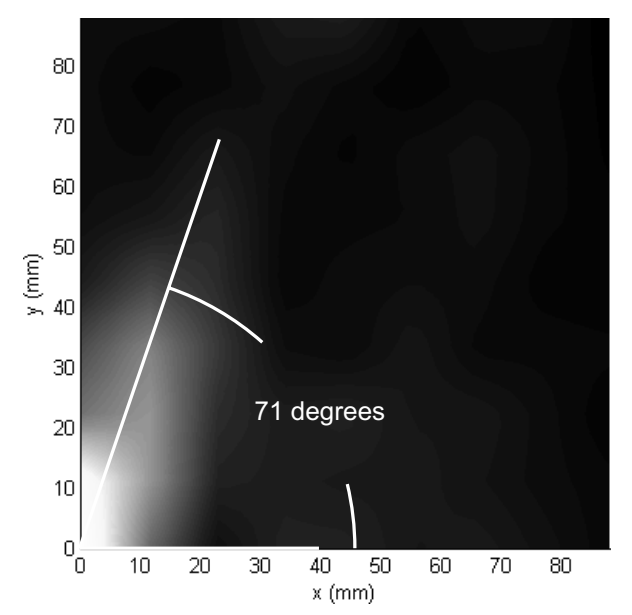

(a) $\mathrm{V}_{\mathrm{R}}=0 \mathrm{~V}$

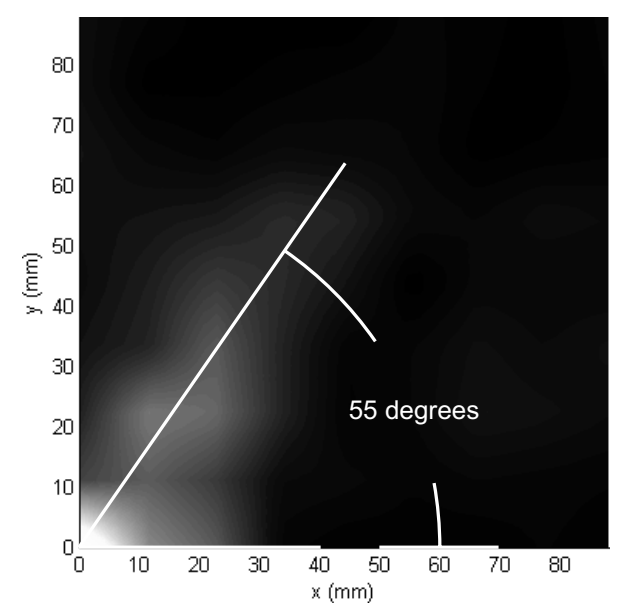

(b) $\mathrm{V}_{\mathrm{R}}=12 \mathrm{~V}$

Figure 3: Near field scans showing the normalized magnitude of the magnetic fields measured across the surface of the ground plane at $1.55 \mathrm{GHz}$.

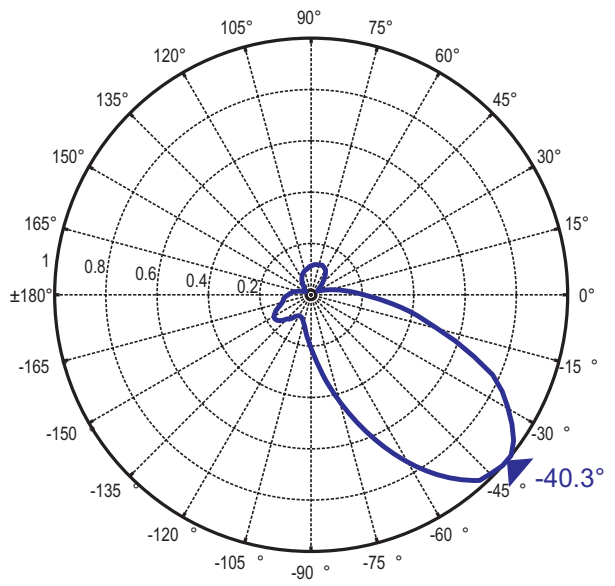

(a) $\mathrm{V}_{\mathrm{R}}=0 \mathrm{~V}$

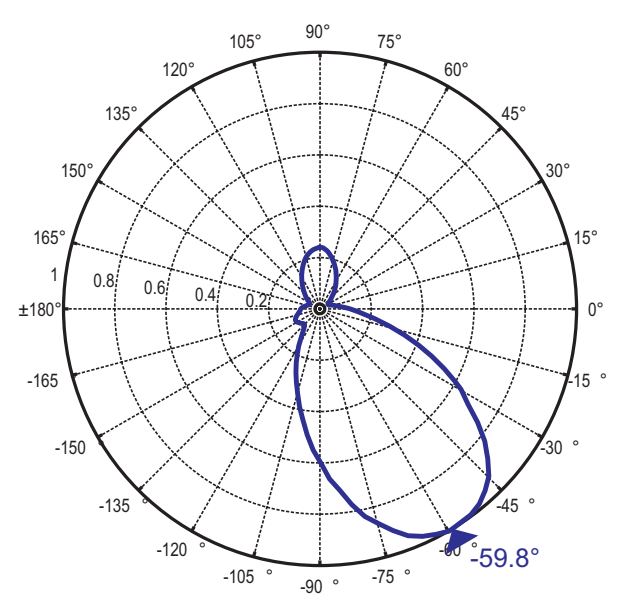

(b) $\mathrm{V}_{\mathrm{R}}=12 \mathrm{~V}$

Figure 4: H-Plane $\left(\theta=90^{\circ}\right)$ normalized power gain as a function of $\varphi$ measured at $1.55 \mathrm{GHz}$.

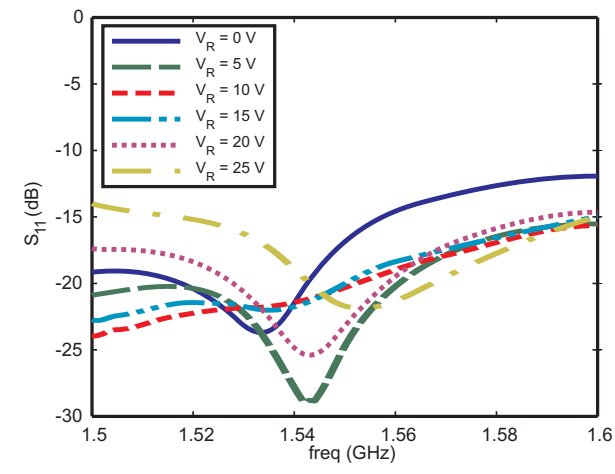

(a)

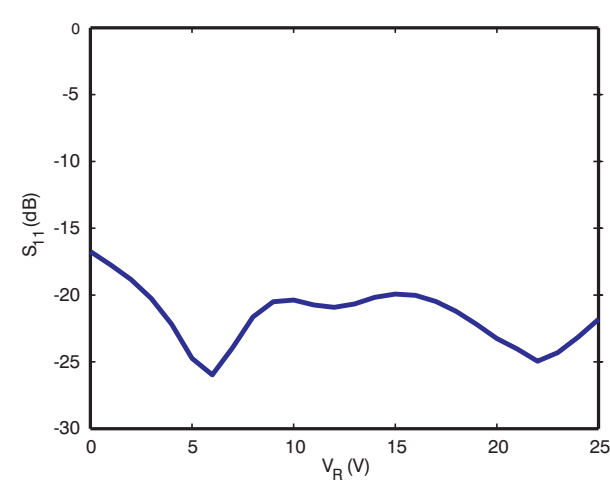

(b)

Figure 5: $S_{11}$ measurements (a) as a function of frequency at various tuning voltages. (b) as a function of tuning voltage at $1.55 \mathrm{GHz}$. 
Another way to visualize the resonance cone is to measure the H-field through the small perforations on the ground plane. A small loop antenna probe, with a loop diameter of $4 \mathrm{~mm}$, is used to probe the H-field as shown in Fig. 2(d). Absorbers were placed around the antenna to prevent any scattering from nearby objects. The antenna is positioned with the ground plane facing toward the probe. The vertical H-field picked up by the probe is fed into a network analyzer measuring the $S_{21}$. The H-field scans, which are normalized with respect to the feed point, are shown in Fig. 3 for a tuning voltage of $0 \mathrm{~V}$ and $12 \mathrm{~V}$ respectively. The plots show the way the resonance cone angle decreases as the tuning voltage is increased. Having confirmed the tunability of the resonance cone angle, the next step is to verify that the far field radiation pattern in the azimuthal plane also follows the same trend. As shown in Fig. 4, the main lobe of the radiation pattern is indeed approximately broadside to the resonance cone and also demonstrates the expected decrease in the main lobe angle at increased tuning voltage. Finally, the characterization is made complete by measuring the return loss for various tuning voltages as shown in Fig. 5 which is better than $16 \mathrm{~dB}$ over the entire tuning range at the design frequency.

\section{Conclusion}

The beam-steerable antenna realized on a tunable hyperbolically anisotropic metamaterial has been constructed and tested. The concept of beam-steering based on spatial filtering has been verified experimentally and shows the expected angular shift in the main beam angle. Unlike a conventional phased array where a feed network is required, the proposed antenna requires only a single RF input and a single bias voltage to adjust the beam direction in the azimuthal plane.

\section{References}

[1] J. K. H. Wong, K. G. Balmain, and G. V. Eleftheriades, "Fields in planar anisotropic transmission-line metamaterials," IEEE Trans. Antennas Propagat., vol. 54, pp. 2742-2749, Oct. 2006.

[2] R. K. Fisher and R. W. Gould, "Resonance cones in the field pattern of a short antenna in an anisotropic plasma," Physical Review Letters, vol. 22, pp. 10931095, May 1969.

[3] K. G. Balmain, A. A. E. Lüttgen, and P. C. Kremer, "Resonance cone formation, reflection, refraction, and focusing in a planar anisotropic metamaterial," IEEE Antennas Wireless Propagat. Lett., vol. 1, pp. 146-149, 2002.

[4] — , "Power flow for resonance cone phenomena in planar anisotropic metamaterials," IEEE Trans. Antennas Propagat., vol. 51, pp. 2612-2618, Oct. 2003.

[5] J. K. H. Wong, K. G. Balmain, and G. V. Eleftheriades, "A diplexer based on the spatial filtering property of planar anisotropic transmission-line metamaterials," in 2006 IEEE International Workshop on Antenna Technology, Mar. 2006, pp. 241-244.

[6] G. V. Eleftheriades and K. G. Balmain, Negative-Refraction Metamaterials: Fundamental Principles and Applications. Wiley-IEEE Press, June 2005. 